\title{
THE HOMOLOGY OF HEISENBERG LIE ALGEBRAS OVER FIELDS OF CHARACTERISTIC TWO
}

\author{
EMIL SKÖLDBERG
}

\begin{abstract}
The generating function of the Betti numbers of the Heisenberg Lie algebra over a field of characteristic 2 is calculated using discrete Morse theory.
\end{abstract}

The Heisenberg Lie algebra of dimension $2 n+1$, denoted by $\mathfrak{h}_{n}$, is the vector space with basis $B=\left\{z, x_{1}, \ldots, x_{n}, y_{1}, \ldots y_{n}\right\}$ where the only non-zero Lie products of basis elements are

$$
\left[x_{i}, y_{i}\right]=-\left[y_{i}, x_{i}\right]=z .
$$

In this paper the Betti numbers of the homology of $\mathfrak{h}_{n}$ over a field of characteristic 2 is computed with the aid of algebraic discrete Morse theory from [Skö]. The notation from [Skö] will be freely used.

Theorem 1. The generating function of the Betti numbers of the Heisenberg Lie algebra over a field of characteristic 2 is

$$
\sum_{i \geq 0} \operatorname{dim}_{k} H_{i}\left(\mathfrak{h}_{n}\right) t^{i}=\frac{\left(1+t^{3}\right)(1+t)^{2 n}+\left(t+t^{2}\right)(2 t)^{n}}{1+t^{2}}
$$

When the ground field of $\mathfrak{h}_{n}$ has characteristic 0, Santharoubane [San83] has shown that

$$
\operatorname{dim}_{k} H_{i}\left(\mathfrak{h}_{n}\right)=\left(\begin{array}{c}
2 n \\
i
\end{array}\right)-\left(\begin{array}{c}
2 n \\
i-2
\end{array}\right),
$$

(the need for the ground field to have characteristic 0 is not explicitly mentioned).

Let us first recall the construction of the Chevalley-Eilenberg complex $\mathbf{V}$ of $\mathfrak{h}_{n}$, whose homology is the homology of $\mathfrak{h}_{n}$ : the complex $\mathbf{V}$ is given by

$$
0 \longrightarrow \bigwedge^{2 n+1} \mathfrak{h}_{n} \longrightarrow \cdots \longrightarrow \bigwedge^{i} \mathfrak{h}_{n} \longrightarrow \cdots \longrightarrow \bigwedge^{2} \mathfrak{h}_{n} \longrightarrow \mathfrak{h}_{n} \longrightarrow 0
$$

with the differential

$$
\bar{d}\left(w_{1} \wedge \cdots \wedge w_{n}\right)=\sum_{i<j}(-1)^{i+j}\left[w_{i}, w_{j}\right] \wedge w_{1} \wedge \cdots \wedge \widehat{w_{i}} \wedge \cdots \wedge \widehat{w_{j}} \wedge \cdots \wedge w_{n}
$$

for $w_{i} \in B$.

The $p$-th homology (with trivial coefficients) of $\mathfrak{h}_{n}$, can now be obtained as the $p$-th homology group of the complex $\mathbf{V}$.

If $I=\left\{i_{1}, \ldots, i_{s}\right\}$ is a subset of $[n]$, we will use the notation $x_{I}$ for the element $x_{i_{1}} \wedge \cdots \wedge x_{i_{s}}$, (and similarly for $y_{I}$ ).

Proof. The result is proved by constructing a Morse matching $M$ on the digraph $G_{\mathbf{V}}$, and showing that when $\pi$ is the projection coming from the splitting homotopy of $M$, we have that $\pi(\mathbf{V})$ has trivial differential.

Date: 18th August 2018.

2000 Mathematics Subject Classification. Primary 17B56.

The author was supported by Marie Curie fellowship HPMD-CT-2001-00079. 
The decomposition of the Chevalley-Eilenberg complex we will use is the obvious; we consider the basis for $\mathbf{V}$ given by $\left\{z \wedge x_{I} \wedge y_{J}, x_{I} \wedge y_{J} \mid I, J \subseteq[n]\right\}$.

Let the matching $M$ consist of the following edges in $G_{\mathbf{V}}$ :

$$
x_{I} \wedge y_{J} \rightarrow z \wedge x_{I \backslash\{k\}} \wedge y_{J \backslash\{k\}}
$$

whenever $\max \left(I^{c} \cap J^{c}\right)<\max (I \cap J)$ and $k=\max (I \cap J)$.

There are now two kinds of unmatched elements: first the elements $z \wedge x_{I} \wedge y_{J}$, with $\max \left(I^{c} \cap J^{c}\right)<\max (I \cap J)$, and then the elements $x_{I} \wedge y_{J}$, with $\max \left(I^{c} \cap J^{c}\right)>$ $\max (I \cap J)$.

When $x_{I} \wedge y_{J} \in M^{+}$, there is exactly one element $z \wedge x_{I^{\prime}} \wedge y_{J^{\prime}}$ with $x_{I} \wedge y_{J} \rightarrow$ $z \wedge x_{I^{\prime}} \wedge y_{J^{\prime}}$ that is not in $M^{0}$, which implies that there can be no directed cycle in the graph $G_{\mathbf{V}}^{M}$. Together with the fact that for all edges in $G_{\mathbf{V}}$ the corresponding component of the differential is an isomorphism, this implies that $M$ is a Morse matching.

We will now see that the differential in $\pi(\mathbf{V})$ is zero. For an element $z \wedge x_{I} \wedge y_{J} \in$ $M^{0}$ it is obvious that $d \pi\left(z \wedge x_{I} \wedge y_{J}\right)=\pi d\left(z \wedge x_{I} \wedge y_{J}\right)=0$. For $x_{I} \wedge y_{J} \in M^{0}$ with $m=\max \left(I^{c} \cap J^{c}\right)$ we get that

$$
\pi\left(x_{I} \wedge y_{J}\right)=x_{I} \wedge y_{J}+\sum_{i \in I \cap J} x_{(I \backslash\{i\}) \cup\{m\}} \wedge y_{(J \backslash\{i\}) \cup\{m\}},
$$

from which it is easily seen that $d \pi\left(x_{I} \wedge y_{J}\right)=0$. From [Skö, Theorem 1] now follows that the $i$-th Betti number is equal to the number of unmatched vertices in homological degree $i$.

For the computation of the generating function we introduce the elements $u_{i}=$ $x_{i} \wedge y_{i}$, and we begin by counting the critical vertices $z \wedge x_{I} \wedge y_{J} \wedge u_{K}$ and $x_{I} \wedge y_{J} \wedge u_{K}$ when $I \cup J=L$ for a fixed set $L \subseteq[n]$.

If $L=[n]$, the critical vertices are all $z \wedge x_{I} \wedge y_{J}$ and $x_{I} \wedge y_{J}$ and they contribute with $(1+t)(2 t)^{n}$ toward the homology.

If $L \neq[n]$, then the critical vertices of the form $z \wedge x_{I} \wedge y_{J} \wedge u_{K}$ are those with $\max ([n] \backslash(I \cup J)) \in K$ so they contribute with $t^{3}(2 t)^{|L|}\left(1+t^{2}\right)^{n-|L|-1}$ toward the homology. The critical vertices of the form $x_{I} \wedge y_{J} \wedge u_{K}$ are those with $\max ([n] \backslash(I \cup J)) \notin K$ and thus contribute with $(2 t)^{|L|}\left(1+t^{2}\right)^{n-|L|-1}$ toward the homology.

Summing up we get

$$
\begin{aligned}
f(t) & =(1+t)(2 t)^{n}+\left(1+t^{3}\right) \sum_{L \subset[n]}(2 t)^{|L|}\left(1+t^{2}\right)^{n-|L|-1} \\
& =(1+t)(2 t)^{n}+\left(1+t^{3}\right) \sum_{i=0}^{n-1}\left(\begin{array}{c}
n \\
i
\end{array}\right)(2 t)^{i}\left(1+t^{2}\right)^{n-i-1} \\
& =(1+t)(2 t)^{n}+\left(1+t^{3}\right)\left(1+t^{2}\right)^{-1}\left(\left(1+2 t+t^{2}\right)^{n}-(2 t)^{n}\right) \\
& =\frac{(1+t)\left(1+t^{2}\right)(2 t)^{n}}{1+t^{2}}+\frac{\left(1+t^{3}\right)(1+t)^{2 n}-\left(1+t^{3}\right)(2 t)^{n}}{1+t^{2}} \\
& =\frac{\left(1+t^{3}\right)(1+t)^{2 n}+\left(t+t^{2}\right)(2 t)^{n}}{1+t^{2}}
\end{aligned}
$$

\section{REFERENCES}

[San83] L. J. Santharoubane, Cohomology of Heisenberg Lie algebras, Proc. Amer. Math. Soc. 87 (1983), no. 1, 23-28. MR 84b:17010

[Skö] Emil Sköldberg, Combinatorial discrete Morse theory from an algebraic viewpint, Preprint, Stockholm University. 
Department of Mathematics, National University of Ireland, Galway, Ireland

E-mail address: emil.skoldberg@nuigalway.ie

URL: www.math.su.se/ ${ }^{\sim e m i l}$ 